\title{
Simultaneous Slack Budgeting and Retiming for Synchronous Circuits Optimization
}

\author{
Shenghua Liu ${ }^{1} \quad{\text { Yuchun } \mathrm{Ma}^{1} \quad \text { Xianlong Hong }}^{1} \quad$ Yu Wang $^{2}$ \\ Tsinghua National Laboratory for Information Science and Technology \\ ${ }^{1}$ Department of Computer Science \& Technology, ${ }^{2}$ Department of Electronic Engineering \\ Tsinghua University, Beijing 100084, China
}

\begin{abstract}
With the challenges of growing functionality and scaling chip size, the possible performance improvements should be considered in the earlier IC design stages, which gives more freedom to the later optimization. Potential slack as an effective metric of possible performance improvements is considered in this work which, as far as we known, is the first work that maximizes the potential slack by retiming for synchronous sequential circuit. A simultaneous slack budgeting and incremental retiming algorithm is proposed for maximizing potential slack. The overall slack budget is optimized by relocating the FFs iteratively with the MIS-based slack estimation. Compared with the potential slack of a well-known min-period retiming, our algorithm improves potential slack averagely $19.6 \%$ without degrading the circuit performance in reasonable runtime. Furthermore, at the expense of a small amount of timing performance, $0.52 \%$ and $2.08 \%$, the potential slack is increased averagely by $19.89 \%$ and $28.16 \%$ separately, which give a hint of the tradeoff between the timing performance and the slack budget.
\end{abstract}

\section{Introduction}

With the coming up of SoC (system on a chip) and SiP (system in a package), more and more devices trend to be put in the small silicon area while at the same time the clock frequency is pushed even higher. Thus timing, area, and power dissipation, which are the three major design objectives, become even more challenging in modern circuit design. Typically, the goal of performance optimization is either to minimize the clock period within a given area and/or power budget [1-3], or to minimize their area and/or power dissipation under timing constraints [4-10]. Particularly, timing budget is often performed in order to slow down as many components as possible without violating the system's timing constraints. The slowed-down components can be further optimized to improve system's area, power dissipation, or other design quality metrics.

For timing-constrained gate-level synthesis, time slack is an effective metric of circuit's potential performance improvement. The slack budgeting problem on a graph has been studied in theory and practice for many different applications, such as timing-driven placement [16-17] and floorplanning [11], gate/wire sizing and power optimization [12-15], and etc. Most of the previous slack budgeting approaches are suboptimal heuristics such as Zero-Slack Algorithm (ZSA) [18]. In [12, 19-20], slack budgeting problem in combinatorial circuit is formulated as maximum-independent-set (MIS) on sensitive transitive closure graph. Recently, [21] presented an LP-based slack budgeting and maximized the potential slack by clock skew optimization. [22] solved the problem of integral delay

This work is supported by NSFC 60606007, 60870001, and 60720106003, 863 project (No. 2009AA01Z130), and TNList Cross-discipline Foundation. budgeting through LP relaxation. [23] proposed combinatorial methods based on net flow approach to handle the slack budget problem. However, the existing slack budgeting algorithms are either used for combinatorial circuit, or limited to fixed FF locations. At the early design stages, there is flexibility to schedule pipeline or timing distribution to obtain more timing slack.

As one of the most powerful sequential optimization techniques, retiming was first proposed by Leiserson and Saxe in 1983 [24], which relocates the flip-flops (FFs) in a circuit while preserving its functionality. The past twenty years saw retiming's effectiveness on improving circuits' timing, area, and power characteristics. Recent publications [1] and [3] proposed a very efficient retiming algorithm for minimal period by algorithm derivation. [2] and [5] later presented efficient incremental algorithms for min-period retiming under setup and hold constraints, and min-area retiming under a given clock period. $[8,25-26]$ proposed the ILP/MILP-based algorithms of retiming for power reduction. Besides, retiming can also increase the potential slack which translates to potential performance. As illustrated in Fig. 1, the period of a circuit is minimized with the delay and slack labeled beside each gate as well. It is seen that there is no potential slack in this circuit. However, if retiming process is taken, i.e. moving the FF from edge $\mathrm{ED}$ to $\mathrm{DC}$, the potential slack can be increased from 0 to 4 , keeping the period minimized at the same time. Though there are massive retiming algorithms, none of them can take slack budget into consideration directly. [28] proposed an LP formulation for optimizing the slack budgeting on interconnects between gates, in order to guide timing-driven placement. However, such an interconnect-based slack budgeting formulation is not easy to transform to gate-based one, since one gate can belong to different interconnects. Furthermore, solving LP formulation with a large problem size is not efficient. In this paper, we propose an incremental retiming approach which can optimize the slack distribution on gates efficiently without any performance degradation.

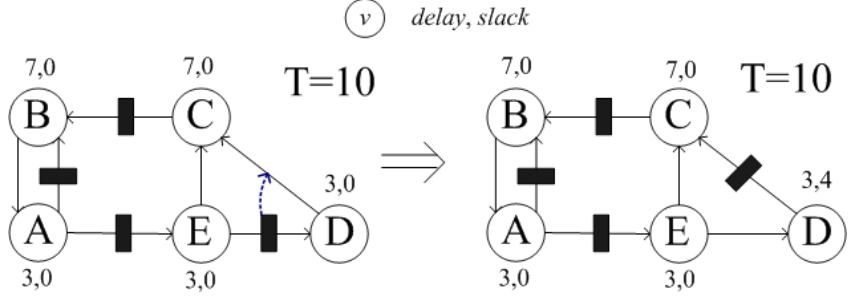

Fig. 1. Relocate a FF to increase potential slack without degrading timing performance

As far as we know, this paper is the first work that maximizes the potential slack by retiming for synchronous sequential circuit. A simultaneously slack budgeting and incremental retiming algorithm is proposed. The formulation 
of MIS on sensitive transitive closure graph is extended from combinatorial circuit to synchronous sequential circuit for potential slack estimation. The overall slack budget can be optimized by relocating the FFs iteratively with the MIS based slack estimation.

Experimental results show that comparing with the potential slack of a well-known min-period retiming [3], our retiming approach increases the potential slack averagely by $19.6 \%$ without degrading timing performance. Meanwhile, retiming and slack budgeting is performed with relaxation of the timing constraint by $2 \%$ and $5 \%$, and the potential slack increases $19.6 \%$ and $28.16 \%$ respectively, which gives a hint to designers about the tradeoff between performance and slack budget or other metrics.

The rest of this paper is organized as follows. Section II introduces the problem formulation. Section III gives three motivating examples of retiming for maximizing potential slack. Sensitive transitive closure graph and slack budgeting for estimating potential slack of synchronous circuit are described in Section IV. Section V presents the simultaneous slack budgeting and retiming algorithm. Experimental results are given in Section VI, and our paper concludes in Section VII.

\section{Preliminaries}

As in [24], we model a synchronous sequential circuit as a directed graph $G=(V, E, d, w)$. Each vertex $v \in V$ represents a combinational gate and each edge $(u, v) \in E$ represents a signal passing from gate $u$ to gate $v$. Non-negative gate delays are given as vertex weights $d: V \rightarrow R^{*}$ and non-negative integer $w: E \rightarrow Z^{*}$ as the edge weight represents the number of FFs on the signal pass. A special host vertex $H$, the edges from host to the primary inputs, and the edges from the primary outputs to host, are introduced into the graph to represent interfaces with the external environment. Without loss of generality, we assume that $G$ is strongly connected. Conventionally, three non-negative labels, $a_{i} / \gamma_{i} / s_{i}: V \rightarrow R^{*}$, represent the latest arrival time, require time, and slack of gate $i . a_{i}$ and $\gamma_{i}$ can be calculated recursively.

$$
\begin{aligned}
& \left\{\begin{array}{lc}
\text { init }: a_{n}=d_{n} & \text { if } w_{m, n}>0 \text { or } n \in F O(H) \\
a_{i}=\max _{j}\left(a_{j}+d_{i}\right) & v_{j} \in F I\left(v_{i}\right)
\end{array}\right. \\
& \left\{\begin{array}{lc}
\text { init }: \gamma_{m}=T & \text { if } w_{m, n}>0 \text { or } m \in F I(H) \\
\gamma_{i}=\min _{k}\left(\gamma_{k}-d_{k}\right) & v_{k} \in F O\left(v_{i}\right)
\end{array}\right.
\end{aligned}
$$

where $w_{m, n}$ means the number of FFs on any directed edge $\left(v_{m}, v_{n}\right)$, and $F I\left(v_{i}\right)$ and $F O\left(v_{i}\right)$ represent the incoming and outgoing gates to gate $v_{i}$ respectively. init is the initialization condition. Slack $s_{i}$ is then calculated by

$$
s_{i}=\gamma_{i}-a_{i}
$$

To represent the reallocation of FFs in retiming, an integer label $r: V \rightarrow \mathrm{Z}^{*}$ is introduced to represent how many FFs are moved from the outgoing edges to the incoming edges of each node. When $r$ is negative value, it means FFs are moved from the incoming edges to outgoing edges. Thus the number of FFs on edge $\left(v_{i}, v_{j}\right)$ with current label $r$ is as formula (4).

$$
w_{i, j}+r_{j}-r_{i}
$$

Our problem is formulated as following.

Problem of simultaneous slack budget and retiming (SSBR): Given a directed graph $G=(V, E, d, w)$ representing a synchronous sequential circuit, and period constraint $T$, we want to find FFs reallocation represented by $r$, such that the potential slack $P_{s}$ obtained by slack budgeting is maximized under the period constraint.

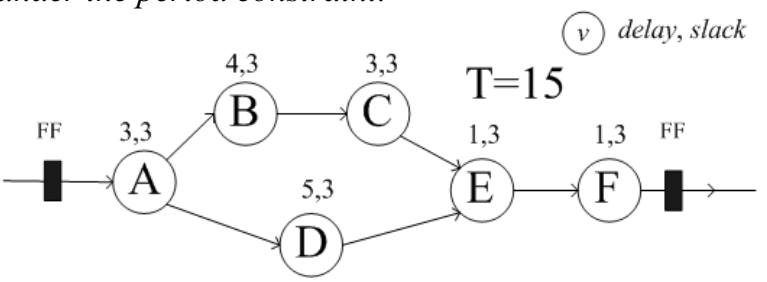

Fig. 2. Slack budgeting in a part of circuit

\section{Motivating Examples}

The potential slack heavily depends on the topology of the graph. In Fig. 2, the delay and slack are labeled beside each gate with clock period $T=15$. When increasing the delay of gate $\mathrm{A}$, the slack of gate $\mathrm{B}$ is reduced as well. Intuitively, if we assign slack to gate A, there are only 3 timing units could be used totally. But gate B and gate D are located on different paths, thus we can increase the delay of gates B and D simultaneously so that total potential slack can be as much as 6 timing units. Thus, slack is needed to be budgeted in order to maximize the potential slack, with which the designer can predict the potential area/power reduction without having to go through actual low-level area/power optimization [19].

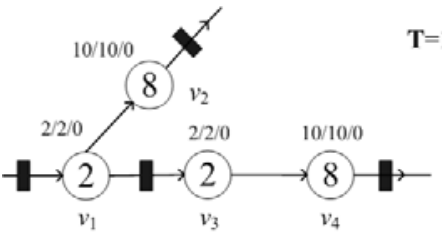

(a)

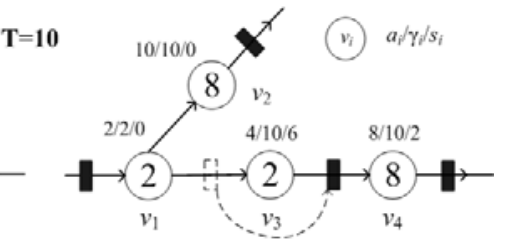

(b)

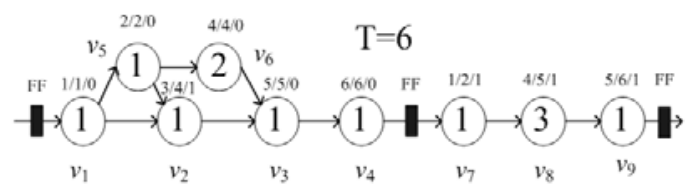

(c)

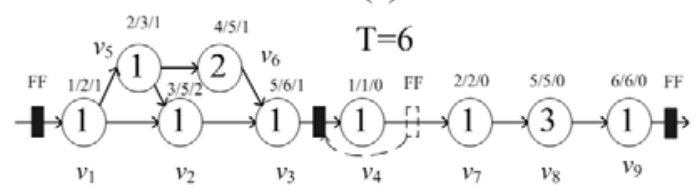

(d)

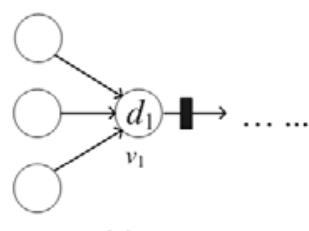

(e)

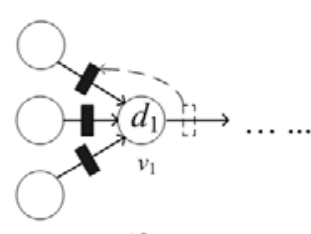

(f)
Fig. 3. Three scenarios for improving potential slack by retiming

As one of the most powerful sequential optimization techniques, retiming has been used to minimize the clock period, area and power. Besides of those benefits, retiming can be used as an effective optimization method to improve the potential slack while preserving circuits' functionality and timing performance. By studying the topological structure of circuits, we find three different scenarios that the potential slack can be improved significantly by relocating 
the FFs.

Scenario I: make use of "slack" on an output. As shown in Fig. 3 (a), there is no slack for each gate. But after further analysis, the signal arrival time of the output edge $\left(v_{1}, v_{3}\right)$ of gate $v_{1}$ is 2 . And this output signal is actually required to arrive at the input of the FF on edge $\left(v_{1}, v_{3}\right)$ before 10 . Thus the timing "slack" on this output is 8 . Since the signal arrival time of the other output $\left(v_{1}, v_{2}\right)$ is required before $\gamma_{2}-d_{2}=2$, according to formulas (2) and (3), the required time on this gate is $\gamma_{1}=2$, and slack is $s_{1}=0$. However, with moving FF from $\left(v_{1}, v_{3}\right)$ to $\left(v_{3}, v_{4}\right)$, the timing "slack" on the output can be used by the gates $v_{3}$ and $v_{4}$, as Fig. 3 (b) shows. So, the total potential slack increased from 0 to 8 .

Scenario II: redistribute the slack to the sub-graph with more branches. The sub-graph here refers to one of the sub-graphs divided by FFs in graph $G$. The branches are actually the independent set, in which vertices are not slack sensitive to each other (Explained in Section IV). As Fig. 3 (c) shows, set $\left\{v_{6}, v_{2}\right\}$ is an independent set in sub-graph of $\left\{v_{1}, v_{2}, v_{3}, v_{5}, v_{6}\right\}$, while the size of independent set in other part is 1 . Thus when moving the FF from edge $\left(v_{4}, v_{7}\right)$ to $\left(v_{3}, v_{4}\right)$ as in Fig. 3 (d), slack is increased from 2 to $s_{2}+s_{6}=3$.

Scenario III: move FF to the gate side with more edges. It is obvious that such a movement increases the slack at least $2 d_{1}$ shown in Fig. 3 (e) and (f). However, such potential slack is increased at the expense of more chip area and power consumed by more FFs. Thus, a combined cost to estimate the real potential performance is given as follows.

$$
\mathcal{P}^{*}=\mathcal{P}-\alpha \Delta n
$$

where $\mathcal{P}$ and $\mathcal{P}^{*}$ are potential slack and combined potential slack, considering the cost of increasing FFs. $\Delta n$ is the increased number of FFs, and constant $\alpha$ reflects the impacts of add one more FFs to the circuits. For the purpose of maximizing the potential slack, $\alpha$ can be assigned to the delay of a FF. And if the chip area is very critical, $\alpha$ can be a very large number, so that Scenario III will not be considered.

It is worth to mention that max-potential-slack retiming may become complicated when there appears any negative-weight edge, after relocating FFs. For such case, we propose a more general method in Section V. Thus, to solve the $S S B R$ problem, it not only needs slack budgeting for various circuit topologies affected by different FF locations, but also needs iteratively retiming to find a optimized FF locations that maximizes potential slack.

\section{Time Slack Budgeting for Estimating Potential Slack}

To estimate the potential slack, a sensitive transitive closure graph is built up for a synchronous circuit. And a MIS-based method is presented for budgeting slack.

\section{A. Sensitive transitive closure graph}

The essential difference between combinatorial circuits and synchronous sequential ones is that the later ones have FFs on signal passes. The edge with FFs cannot be slack sensitive because the signals on the two sides of FFs are in different stage of sequential circuits. Thus, we could easily borrow the concepts of slack sensitive from combinatorial circuit with some modifications. With the help of the labels $a / \gamma / s$ and $r$ on each vertex of graph $G(V, E, d, w)$, we formally define edge $\left(v_{i}, v_{j}\right) \in E$ to be slack sensitive if and only if the condition (6) is satisfied.

$$
w_{i j}+r_{j}-r_{i}=0 \text { and }\left(a_{j}-a_{i}=d_{j} \text { or } \gamma_{j}-\gamma_{i}=d_{j}\right)
$$

where $w_{i j}+r_{j}-r_{i}=0$ means there is no FF on edge $\left(v_{i}, v_{j}\right)$. From formula (1), the edge with $a_{j}-a_{i}=d_{j}$ is a sensitive edge, on which the arrival time $a_{j}=a_{i}+d_{j}$ of gate $v_{i}$ actually depends. In the same way, from formula (2), the edge with $\gamma_{j}$ $-\gamma_{i}=d_{j}$ is also sensitive, since the required time $\gamma_{i}=\gamma_{j}-d_{j}$ of gate $v_{i}$ depends on it. According to the definition, for the example in Fig. $3(\mathrm{~d})$, edge $\left(v_{1}, v_{2}\right)$ is not slack sensitive $\left(r_{1}=\right.$ $r_{2}=0, a_{2}-a_{1}>d_{1}$ and $\left.\gamma_{2}-\gamma_{1}>d_{1}\right)$, while edge $\left(v_{5}, v_{2}\right)$ is slack sensitive, $\left(r_{2}=r_{5}=0, a_{2}-a_{5}=d_{5}\right)$. The sensitive transitive closure graph (STCG) is $G_{s}\left(V, E_{s}\right)$ of $G$ is a directed graph such that there is an edge $\left(v_{i}, v_{j}\right)$ in $G_{s}$ if and only if there is a directed path from $v_{i}$ to $v_{j}$ in $G$ and the path consists of only sensitive edges. Two vertices $v_{i}$ and $v_{j}$ are called slack insensitive if there is no edge from $v_{i}$ to $v_{j}$ or from $v_{j}$ to $v_{i}$ in $G_{s}$. A set $S I=\left\{v_{1}, v_{2}, \ldots, v_{k}\right\}$ of vertices is called slack insensitive if all vertices in the set are pair-wise slack insensitive. Let each edge in $G_{s}$ replaced by an undirected edge, and become an undirected graph $\bar{G}_{s}$. So, the slack insensitive set $S I$ is an independent set of graph $\bar{G}_{S}$. Fig. 4 (a) highlights the slack sensitive edges in Fig. 3 (d) with dotted lines. And then the undirected STCG is given in Fig. 4 (b) which consists of two connected sub-graphs.

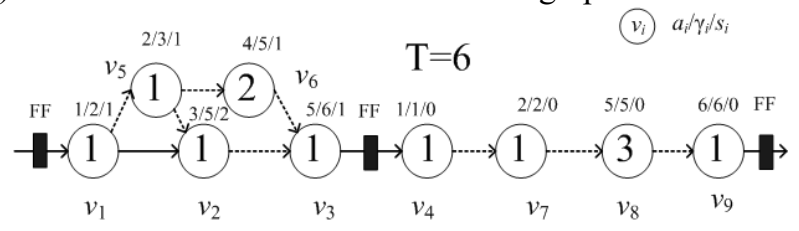

(a)
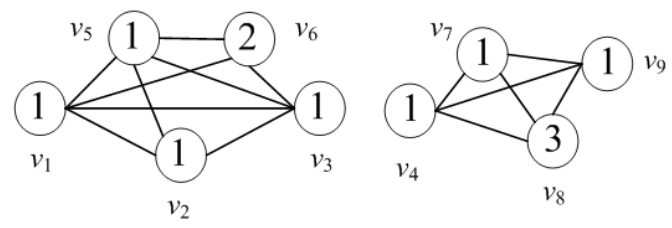

(b)

Fig. 4. Slack sensitive edges with dot lines, (b) is the undirected STCG of (a)

\section{B. Estimating Potential Slack (EPS)}

After labeling $a / \gamma / s$, we collect all the vertices with positive slack into set $Q$. An induced graph of $\bar{G}_{s}$ on $Q$ is named as $\bar{G}_{q}$. And let a maximum independent set of $\bar{G}_{q}$ be $S I$. Then, each vertex in set $Q$ can increase its delay by $\mathcal{S}=$ $\min \left\{s_{i} \mid v_{i} \in S I\right\}$ separately without breaking the clock period constraint. And then we iteratively find the set SI, and increase delay until $Q$ is empty. At last, the potential slack is the summation of the increased delay in every iteration. The algorithm of potential slack budgeting is given in Fig. 5. The optimality of the algorithm was proved in [12]. And a well-know polynomial-time MIS solver by Dharwadker [27] is employed in step 4 of Fig. 5, which solves the MIS problem by the duality with minimum vertex cover.

We still take the example in Fig. 4 to illustrate the process. The induced graph of $\bar{G}_{S}$ on $\left\{v_{1}, v_{2}, v_{3}, v_{5}, v_{6}\right\}$ is the left connected sub-graph. Thus the maximum independent set $S I$ is $\left\{v_{2}, v_{6}\right\}, \mathcal{S}=1$, and $p_{s}=2$. After increasing the delay of each vertex, $\bar{G}_{S}$ contains only vertex $v_{2}$ with slack $s_{2}=1$. Thus $S I$ 
$=\left\{v_{2}\right\}, \mathcal{S}=1$ and $p_{s}=2+\mathcal{S}=3$. Afterwards, the subroutine terminates and successfully output the budgeted slack $p_{s}=3$.

1. $p_{s}=0$;

2. let vertices with positive slack be $Q$;

3. While ( $Q$ is nonempty) \{

4. build induced graph of $\bar{G}_{S}$ on $Q$;

5. find maximum independent set $S I$;

6. $\mathcal{S}=\min \left\{s_{i} \mid v_{i} \in S I\right\}$

7. $p_{s}+=\mathcal{S} * \operatorname{sizeof}(S I)$;

8. Foreach $v_{i} \in Q \quad\left\{d_{i}+=\mathcal{S} ;\right\}$

9. update $a / \gamma / s$ and $Q$;

10. $\}$

11. recover delay $d$ of graph $G$;

12. output potential slack $p_{s}$;

Fig. 5. Subroutine for estimating potential slack (EPS)

\section{Simultaneous Retiming and Slack Budgeting}

\section{A. General form for SSBR}

In section III, we enumerate three scenarios that improve the potential slack by relocating FFs. However, for the case shown in Fig. 3 (a), if retiming vertex $v_{1}$ with labeling $r_{1}=1$ without considering clock period (Fig.6(a)), the new weight of edge $\left(v_{1}, v_{2}\right)$ is $w_{12}+r_{2}-r_{1}=-1$, according to formula (4). It means that the edge $\left(v_{1}, v_{2}\right)$ needs to borrow a FF from outgoing edges of vertex $v_{2}$, in order to legalize the retiming. Thus with $r_{2}=1$, the retiming result is as Fig. 6(b) shows. This procedure is called retiming legalization. Such retiming legalization usually causes the relocation of the FFs in other part of the circuit which may in turn reflect the slack distribution. So, in this section, a more general method is proposed to increase potential sack with retiming under the specific period constraint.

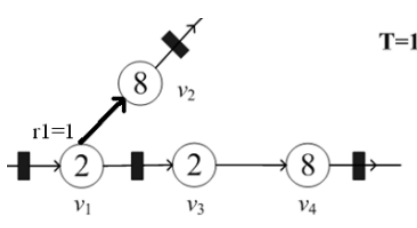

(a)

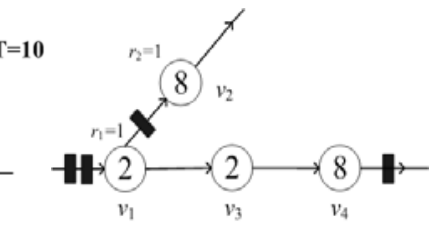

(b)
Fig. 6. (a) Retiming $v_{1}$ with labeling $r_{1}=1$; (b) retiming legalization

Thus simultaneous slack budgeting and retiming can be expressed in the following form.

$$
\begin{gathered}
\text { Max } p_{s} \\
\text { s.t. } \quad w_{i, j}+r_{j}-r_{i} \geq 0, \forall\left(v_{i}, v_{j}\right) \in E \\
a_{i} \leq T, \forall v_{i} \in V
\end{gathered}
$$

Formula (8) shows the constraints that the number of FFs on each edge should not be negative. Formula (9) constrains that the delay of critical path must satisfy the timing constraint $T$.

\section{B. Incremental retiming with possible moves}

Therefore, we can relocate the positions of FFs iteratively without violating the timing constraint and estimate the potential slack for each relocation to find an optimal one. Each step is triggered by a FF relocation which satisfies the timing constraints. Let such an FF relocation be a movement $m$ represented by label $r$. Firstly, we define movement $m_{i}$ over vertex $v_{i}$ is positive if only if it moves FFs from outgoing edges $F O\left(v_{i}\right)$ of $v_{i}$ to its incoming edges $F I\left(v_{i}\right)$, and the reverse movement is negative. If the movement $m_{i}$ is positive, the delay $d_{i}$ of $v_{i}$ will be added to the timing path starting with gate $v_{j} \in F O\left(v_{i}\right)$. Thus, in order to keep condition (10) satisfied, it is necessary that the slack $s_{j}$ of gate $v_{j}$ must be no smaller than the delay $d_{i}$. Hence, condition (10) must be satisfied.

$$
\gamma_{j}-d_{j}-d_{i} \geq 0, \quad v_{j} \in F O\left(v_{i}\right)
$$

In the same way, if the movement $m_{i}$ is negative, the delay $d_{i}$ of $v_{i}$ will be added to the timing path ending with gate $v_{j} \in F I\left(v_{i}\right)$. To keep condition (9) satisfied, it is necessary that the arrival time $a_{j}$ of gate $v_{j}$ plus the delay $d_{i}$ must not exceed the period $T$. That is condition (11) must be satisfied.

$$
a_{j}+d_{i} \leq T, \quad v_{j} \in F I\left(v_{i}\right)
$$

So, we can collect the possible FF movements $M$ with conditions (10) or (11) satisfied. If condition (10) is satisfied, the next movement $m_{i} \in M$ is possible and represented by label $r$ as $m_{i}=r_{i}+1$, otherwise if condition (11) is satisfied, it is $m_{i}=r_{i}-1$. Although conditions (10) and (11) can reduce much searching space, they are insufficient for condition (8), so some of them have to be picked out after retiming legalization and arrival time calculation, which are discussed later.

When relocating FFs by positive movement $m_{i} \in M$, some outgoing edges may become negative weighted. As described in subsection $A$, the retiming legalization may borrow the FFs from the outgoing edges of the succeeding gates in the directed path. It is practiced recursively by invoking formula (12), until all edges weight are positive.

$$
r_{j}=r_{i}-w_{i j}, v_{j} \in F O\left(v_{i}\right), \text { if } w_{i j}+r_{j}-r_{i}<0
$$

In the same way, when relocating FFs by negative movement $m_{i}$, if there occur negative weighted edges, we legalize the retiming by borrowing the FFs from the incoming edges of the preceding gates. And the retiming legalization is based on formula (13) recursively.

$$
r_{j}=r_{i}+w_{j i}, v_{j} \in F I\left(v_{i}\right), \text { if } w_{j i}+r_{i}-r_{j}<0
$$

After the retiming legalization, the arrival time of each gate is calculated by formula (1). If condition (9) is not satisfied, such movement $m_{i}$ will not be considered. If satisfied, an incremental retiming is performed based on the movement $m_{i}$.

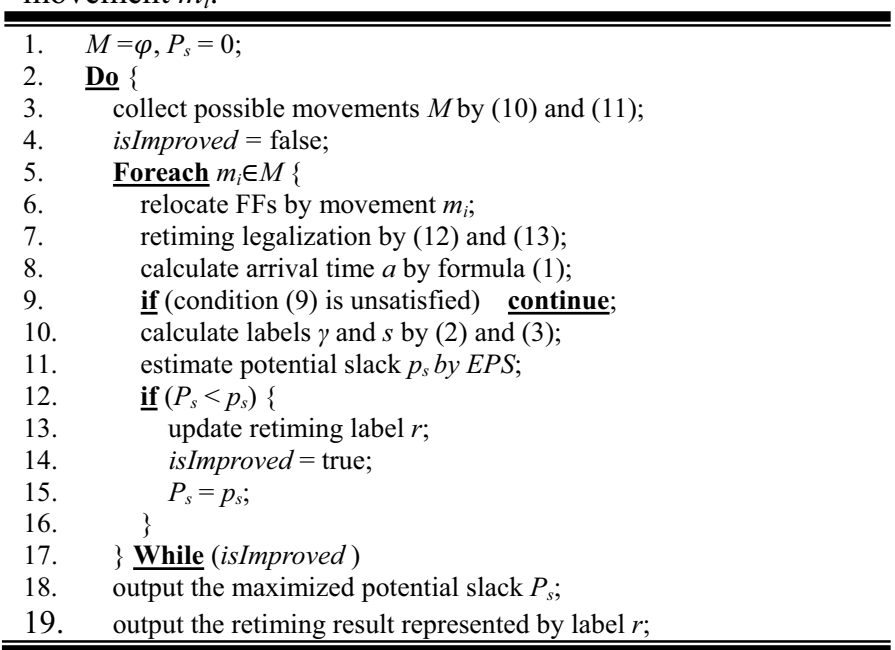

Fig. 7. The algorithm of simultaneous slack budgeting and retiming

C. Iterative incremental retiming flow

The subroutine of EPS is called to estimate potential slack after each incremental retiming with those considered 
movements in $M$. Only the movement that improves the potential slack is accepted, and then retiming label $r$ is updated. The algorithm iteratively collects possible FF movements $M$, and takes the movement that can increase potential slack. The algorithm terminates until there are no potential slack increase any more, and its flow is illustrated in Fig. 7.

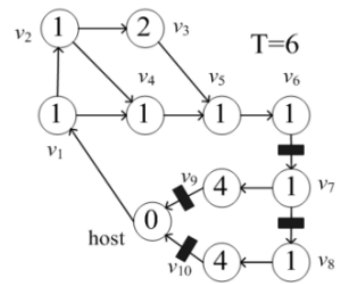

(a)

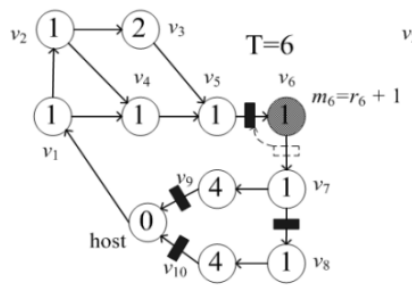

(b)

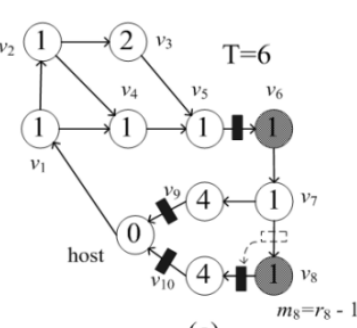

(c)
Fig. 8. An example of our incremental retiming and slack budgeting algorithm. The movements are labeled besides related vertex shown in (b) and (c).

An example in Fig. 8 is used to clarify our idea of incremental retiming for maximizing potential slack. Detailed execution results are listed in the following table step by step, where $M$ is the movements set represented by $r$, $S I$ is the maximum independent set of $\bar{G}_{S}$ with positive slack vertices, and $P_{s}$ is the potential slack. At last, the potential slack increases from 3 to 8 .

\begin{tabular}{|l|l|l|}
\hline & $M, S I, P_{s}$ & comments \\
\hline 1 & $\varphi,\left\{v_{4}, v_{7}, v_{8}\right\}, 3$ & $r_{i}=0, v_{i} \in V$ \\
\hline 2 & $\left\{r_{6}+1, r_{8}-1\right\},\left\{v_{4}, v_{7}, v_{8}\right\}, 3$ & after step 3 in Fig. 7 \\
\hline 3 & $\left\{r_{6}+1, r_{8}-1\right\},\left\{v_{3}, v_{4}, v_{8}\right\}, 4$ & taking movement $r_{6}+1$, and $r_{6}=1$ \\
\hline 4 & $\left\{r_{6}+1, r_{8}-1\right\},\left\{v_{3}, v_{4}, v_{8}, v_{10}\right\}, 8$ & taking movement $r_{6}+1$, and $r_{6}=1, r_{8}=-1$ \\
\hline 5 & $\left\{r_{6}-1, r_{8}+1\right\},\left\{v_{3}, v_{4}, v_{8}, v_{10}\right\}, 8$ & after step 3 in Fig. 7 \\
\hline 6 & $\varphi,\left\{v_{3}, v_{4}, v_{8}, v_{10}\right\}, 8$ (optimal) & $\begin{array}{l}\text { condition (10) is unsatisfied, and } \\
\text { isImproved is false. }\end{array}$ \\
\hline
\end{tabular}

\section{Experimental Results}

The algorithm is implemented with $\mathrm{C}++$, and tested under linux server with eight $3.0-\mathrm{GHz}$ cores and $6 \mathrm{G}$ memories. 19 cases from the ISCAS 89 benchmarks are tested, and the name, number of gates, number of signal passes, the maximum number of gate outputs/inputs, and the minimum period for each case are separately given in Table 1.

In the experiments, a min-period retiming algorithm [3] is employed to get the minimum period of the circuits, and potential slacks are estimated under current fixed FFs, which are listed in the 2 nd and 3 rd column of TABLE II. The simultaneous slack budgeting and retiming are firstly performed on those test cases within min-period timing constraint. The maximized potential slack, the increase ratio of potential slack, and runtime are given separately. The average increase ratio of potential slack is $19.6 \%$ within timing constraint of the min-period $T_{\min }$ in reasonable runtime. And then we relax the timing constraint and perform the algorithm within timing constraint of $(1+2 \%)$
$T_{\min }$ and $(1+5 \%) T_{\min }$. Since the clock period may not reach the bound of the timing constraint, the actual clock period after retiming, the potential slack, the slack increase ratio compare with $P_{0}$, and runtime are given separately for both experiments with timing constraint relaxation in TALBE II. The average increase ratios are $19.89 \%$ and $28.16 \%$ with a little sacrifice of timing performance (increasing the average period by $0.52 \%$ and $2.08 \%$ ), which can give designers some hints to the tradeoff between fast and low-power circuits. Fig. 9 illustrates a direct comparison between $P_{0}$ and the maximized potential slack within three different timing constraints which is normalized by being divided by $P_{0}$. Since the room of slack optimization with retiming depends on the topology of circuit greatly, the improvement varies from 0 to $214.29 \%$. As for s27.test, the circuit size is small, so the increase ratio appears relatively high. We can see that in addition to s27.test, some cases also achieve much improvement, such as $58.76 \%$ and $20.88 \%$, while there is no improvement at all for some others. Furthermore, the relaxed timing constraints may even not influence slack budget. Averagely, we can obtain about 9\% extra slack budget $(19.6 \%$ vs. $28.16 \%)$ with only $2 \%$ performance degradation $(80.95$ vs. 82.63).

TABLE I

The Characteristics of Test cases

\begin{tabular}{c|ccccc}
\hline Test case name & $\begin{array}{c}\text { Gates } \\
\text { count }\end{array}$ & $\begin{array}{c}\text { Edges } \\
\text { count }\end{array}$ & $\begin{array}{c}\text { Max } \\
\text { outputs }\end{array}$ & $\begin{array}{c}\text { Max } \\
\text { inputs }\end{array}$ & $T_{\min }$ \\
\hline s27.test & 11 & 19 & 4 & 2 & 20 \\
s208.1.test & 105 & 182 & 19 & 4 & 28 \\
s298.test & 120 & 250 & 13 & 6 & 24 \\
s382.test & 159 & 312 & 21 & 6 & 44 \\
s386.test & 160 & 354 & 36 & 7 & 64 \\
s344.test & 161 & 280 & 12 & 11 & 46 \\
s349.test & 162 & 284 & 12 & 11 & 46 \\
s444.test & 182 & 358 & 22 & 6 & 46 \\
s526.test & 194 & 451 & 13 & 6 & 42 \\
s526n.test & 195 & 451 & 13 & 6 & 42 \\
s510.test & 212 & 431 & 28 & 7 & 50 \\
s420.1.test & 219 & 384 & 31 & 4 & 40 \\
s832.test & 288 & 788 & 107 & 19 & 98 \\
s820.test & 290 & 776 & 106 & 19 & 92 \\
s641.test & 380 & 563 & 35 & 24 & 238 \\
s713.test & 394 & 614 & 35 & 23 & 262 \\
s838.1.test & 447 & 788 & 55 & 4 & 80 \\
s1238.test & 509 & 1055 & 192 & 14 & 110 \\
s1488.test & 654 & 1406 & 56 & 19 & 166 \\
\hline
\end{tabular}

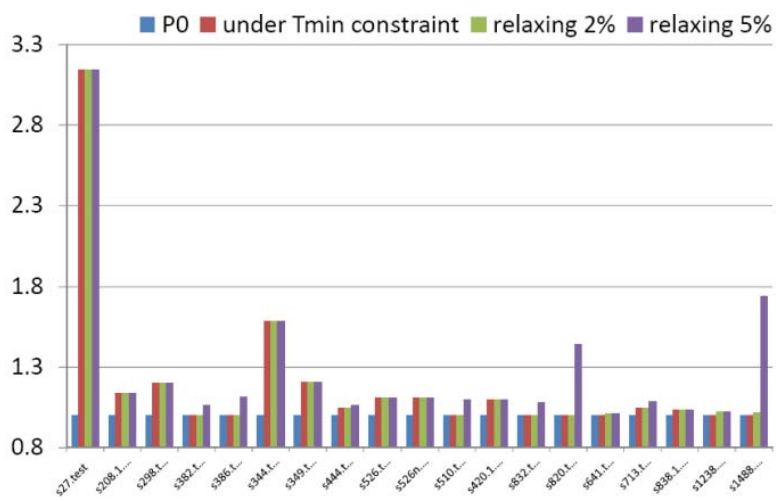

Fig. 9. Normalized $P_{0}$ and the optimized potential slack under three different timing constraints.

\section{Conclusions}

In this work, we introduce the slack sensitive closure graph in combinatorial circuits to synchronous sequential circuits, on which a MIS-based slack budgeting is performed. 
And a simultaneously incremental retiming and slack budgeting algorithm, i.e. max-potential-slack retiming is presented, which is heuristic yet effective. Comparing with the potential slack obtained by a min-period retiming [3], the experimental results show that our algorithm can improve the potential slack averagely by $19.6 \%$ without degrading timing performance in reasonable runtime. Meanwhile, we also perform max-potential-slack retiming with timing constraint relaxation of $2 \%$ and $5 \%$, and the potential slack increases $19.6 \%$ and $28.16 \%$, which gives a view of tradeoff between a fast and low-power design. Since potential slack is a good prediction for potential performance in the gate-level syntheses [19], the retiming for power reduction maximization is our future work to practice its effectiveness.

\section{References}

[1] H. Zhou, "Deriving a New Efficient Algorithm for Min-Period Retiming," in Proc. Asia South Pacific Design Automation Conf., pp.990-993, 2005.

[2] C. Lin and H. Zhou, "An efficient retiming algorithm under setup and hold constraints," in Proc. of IEEE/ACM Design Automation Conf., pp.945-950, 2006.

[3] H. Zhou, "A New Efficient Retiming Algorithm Derived by Formal Manipulation," ACM Trans. Design Automation of Electronics Systems, Vol. 13(1), 2008.

[4] N. Maheshwari and S. Sapatnekar, "An improved algorithm for minimum-area retiming," in Proc. of Design Automation Conf., pp.2-7, 1997.

[5] J. Wang, H. Zhou, "An efficient incremental algorithm for min-area retiming," in Proc. of Design Automation Conf., pp.528-533, 2008.

[6] J. Monteiro, S. Devadas, and A. Ghosh, "Retiming sequential circuits for low power," in Proc. Int. Conf. Computer-Aided Design, Nov. 1993, pp. 398-402.

[7] K. Lalgudi and M. Papaefthymiou. Fixed-phase retiming for low power. In Proc. Int. Symp. on Low Power Electronics and Design, pp. 259-264, 1996.

[8] N. Chabini, I. Chabini, E. M. Aboulhamid, and Y. Savaria, "Unification of Basic Retiming and Supply Voltage Scaling to Minimize Dynamic Power Consumption for Synchronous Digital Designs," In Proc. Great Lakes Symposum on VLSI, pages 221-224, 2003.

[9] N. Chabini and W.Wol, "Reducing Dynamic Power Consump- tion in Synchronous Sequential Digital Designs Using Retiming and Supply Voltage Scaling,” IEEE Trans. on VLSI Systems, 12(6):573-589, June 2004 [10] M. Ekpanyapong and S. K. Lim, "Integrated retiming and simultaneous Vdd/Vth scaling for total power minimization," in Proc. Intl. Symp. Physical Design, pp. 142-148, 2006.

[11] T. Kong and X. L. Hong, "Timing-driven floor-planning algorithm for building block layout," in Proc. SPIE Vol. 2644, p. 477-482, 1996.
[12] D. S. Chen and M. Sarrafzadeh, "An exact algorithm for low power library-specific gate re-sizing," in Proc. of IEEE/ACM Design Automation Conf., Las Vegas, pp.783 - 788, 1996.

[13] S. Augsburger and B. Nikolic. "Reducing Power with Dual Supply, Dual Threshold and Transistor Sizing," In Proc. IEEE Int. Conf. on Computer Design, pp. 316-321, 2002.

[14] A. Srivastava, D. Sylvester, and D. Blaauw, "Power minimization using simultaneous gate sizing, dual-Vdd and dual-Vth assignment," In Proc. ACM Design Automation Conf., pp.783-787, 2004.

[15] X. Qiu, Y. Ma, X. He, and X. Hong, "IPOSA: A Novel Slack Distribution Algorithm for Interconnect Power Optimization," in Proc. of Intl. Symp. on Quality Electronic Design, pp.873-876, 2008.

[16] Chao-Yang Yeh, Malgorzata Marek-Sadowska, "Delay budgeting in sequential circuit with application on FPGA placement," in Proc. of IEEE/ACM Design Automation Conf. pp.202-207, 2003.

[17] A. P. Hurst, P. Chong, and A. Kuehlmann, "Physical placement driven by sequential timing analysis," in Proc. Int. Conf. Computer-Aided Design, pp. $379-386,2004$

[18] R. Nair, C. L. Berman, P. S. Hauge, and E. J. Yoffa, "Generation of Performance Constraints for Layout," IEEE Transactions on ComputerAided Design, CAD-8(8): 860-874, August 1989.

[19] C. Chen, X. Yang, and M. Sarrafzadeh, "Predicting potential performance for digital circuits," IEEE Trans. Computer-Aided Design, vol. 21(3), pp. 253 - 262, 2002.

[20]C. Chen, E. Bozorgzadeh, A. Srivastava, M. Sarrafzadeh: Budget Management with Applications. Algorithmica 34(3), pp.261-275, 2002.

[21] K. Wang and M. Marek-Sadowska, "Potential Slack Budgeting with Clock Skew Optimization," in Proc. Int. Conf. Computer-Aided Design, pp. 265- 271, 2004.

[22]E. Bozorgzadeh, $S$. Ghiasi, A. Takahashi and M. Sarrafzadeh, "Optimal Integer Delay Budgeting on Directed Acyclic Graphs". in Proc. of IEEE/ACM Design Automation Conf., pp. 920 - 925, 2003.

[23]S. Ghiasi, E. Bozorgzadeh, P. K. Huang, R. Jafari, M. Sarrafzadeh, “A Unified Theory of Timing Budget Management," IEEE Trans. on CAD of Integrated Circuits and Systems 25(11), pp.2364-2375 , 2006.

[24] C. E. Leiserson and J.B. Saxe, "Retiming Synchronous circuitry," Algorithmica, 6(1), pp.5-35, 1991

[25] F. Sheikh, A. Kuehlmann, and K. Keutzer. Minimum-power retiming for dual-supply CMOS circuits. In Proc. of the 8th ACM/IEEE Workshop on Timing issues in the specification and synthesis of digital systems, pp. $43-49,2002$.

[26] Y. Hu, Y. Lin, L. He and T. Tuan, "Simultaneous Time Slack Budgeting and Retiming for Dual-Vdd FPGA Power Reduction," in Proc. of IEEE/ACM Design Automation Conf., pp. 478-483, 2006.

[27] A. Dharwadker, "The Independent Set Algorithm," http://www.geocities.com/dharwadker/independent_set/, 2006.

[28] C.Y. Yeh, and M. Marek-Sadowska, "Sequential Delay Budgeting with Interconnect Prediction," IEEE Trans. on VLSI, 12(10), pp.1028-1037, 2004.

TABEL II

Experimental Results

$T_{\min }$ - the minimum period of the circuit by Zhou's min-period retiming [3]; $P_{0}$ - the potential slack after the min-period retiming

Under $T_{\min }$ constraint - max-potential-slack retiming with timing constraint $T_{\min }$; Relaxing $x \%$-- max-potential-slack retiming with timing constraint $T_{\min } \times(1+x \%)$ period - the actual clock period after under max-potential-slack retiming with timing relaxation

\begin{tabular}{|c|c|c|c|c|c|c|c|c|c|c|c|c|c|}
\hline \multirow{2}{*}{$\begin{array}{c}\text { Test } \\
\text { cases }\end{array}$} & \multicolumn{2}{|c|}{ Zhou's [3] } & \multicolumn{3}{|c|}{ Under $T_{\min }$ constraint } & \multicolumn{4}{|c|}{ Relaxing $2 \%$} & \multicolumn{4}{|c|}{ Relaxing 5\% } \\
\hline & $T_{\min }$ & $P_{0}$ & $P_{s}$ & increase & runtime $(s)$ & period & $P_{s}$ & increase & runtime $(s)$ & period & $P_{s}$ & increase & runtime $(s)$ \\
\hline s27.test & 20 & 14 & 44 & $214.29 \%$ & 0.00 & 20 & 44 & $214.29 \%$ & 0.00 & 20 & 44 & $214.29 \%$ & 0.00 \\
\hline s208.1.test & 28 & 422 & 480 & $13.74 \%$ & 1.30 & 28 & 480 & $13.74 \%$ & 1.33 & 28 & 480 & $13.74 \%$ & 1.30 \\
\hline s298.test & 24 & 522 & 628 & $20.31 \%$ & 6.64 & 24 & 628 & $20.31 \%$ & 6.66 & 24 & 628 & $20.31 \%$ & 6.37 \\
\hline s382.test & 44 & 1204 & 1204 & $0.00 \%$ & 11.58 & 44 & 1204 & $0.00 \%$ & 10.25 & 46 & 1282 & $6.48 \%$ & 5.76 \\
\hline s386.test & 64 & 724 & 724 & $0.00 \%$ & 1.11 & 64 & 724 & $0.00 \%$ & 1.01 & 66 & 806 & $11.33 \%$ & 4.42 \\
\hline s344.test & 46 & 742 & 1178 & $58.76 \%$ & 8.19 & 46 & 1178 & $58.76 \%$ & 7.87 & 46 & 1178 & $58.76 \%$ & 8.21 \\
\hline s349.test & 46 & 728 & 880 & $20.88 \%$ & 7.72 & 46 & 880 & $20.88 \%$ & 8.34 & 46 & 880 & $20.88 \%$ & 6.34 \\
\hline s444.test & 46 & 1208 & 1264 & $4.64 \%$ & 33.55 & 46 & 1264 & $4.64 \%$ & 39.65 & 48 & 1284 & $6.29 \%$ & 13.92 \\
\hline s526.test & 42 & 1324 & 1472 & $11.18 \%$ & 27.08 & 42 & 1472 & $11.18 \%$ & 23.49 & 42 & 1472 & $11.18 \%$ & 13.19 \\
\hline s526n.test & 42 & 1306 & 1446 & $10.72 \%$ & 57.80 & 42 & 1446 & $10.72 \%$ & 71.41 & 42 & 1446 & $10.72 \%$ & 11.81 \\
\hline s510.test & 50 & 1224 & 1224 & $0.00 \%$ & 12.21 & 50 & 1224 & $0.00 \%$ & 11.62 & 52 & 1342 & $9.64 \%$ & 25.60 \\
\hline s420.1.test & 40 & 1318 & 1444 & $9.56 \%$ & 42.68 & 40 & 1444 & $9.56 \%$ & 41.99 & 40 & 1444 & $9.56 \%$ & 40.02 \\
\hline s832.test & 98 & 6130 & 6130 & $0.00 \%$ & 61.01 & 98 & 6130 & $0.00 \%$ & 42.51 & 102 & 6618 & $7.96 \%$ & 462.27 \\
\hline s820.test & 92 & 5746 & 5746 & $0.00 \%$ & 42.24 & 92 & 5746 & $0.00 \%$ & 48.18 & 92 & 8302 & $44.48 \%$ & 503.07 \\
\hline s641.test & 238 & 9624 & 9624 & $0.00 \%$ & 329.63 & 242 & 9758 & $1.39 \%$ & 441.96 & 242 & 9758 & $1.39 \%$ & 1979.38 \\
\hline s713.test & 262 & 11234 & 11760 & $4.68 \%$ & 431.90 & 262 & 11760 & $4.68 \%$ & 504.08 & 270 & 12174 & $8.37 \%$ & 483.73 \\
\hline s838.1.test & 80 & 7038 & 7292 & $3.61 \%$ & 1674.93 & 80 & 7292 & $3.61 \%$ & 1840.07 & 80 & 7292 & $3.61 \%$ & 1075.06 \\
\hline s1238.test & 110 & 9260 & 9260 & $0.00 \%$ & 3318.06 & 112 & 9462 & $2.18 \%$ & 2178.99 & 112 & 9462 & $2.18 \%$ & 8626.44 \\
\hline s1488.test & 166 & 11436 & 11436 & $0.00 \%$ & 884.97 & 168 & 11662 & $1.98 \%$ & 2843.89 & 172 & 19880 & $73.84 \%$ & 6255.33 \\
\hline Average & 80.95 & & & $19.6 \%$ & 365.93 & $81.37(+0.52 \%)$ & & $19.89 \%$ & 427.54 & $82.63(+2.08 \%)$ & & $28.16 \%$ & 1027.49 \\
\hline
\end{tabular}

